\title{
Role of computed tomography features in the differential diagnosis of chromophobe renal cell carcinoma from oncocytoma and angiomyolipoma without visible fat
}

\author{
Cuiping Zhou ${ }^{1 \#}$, Xiaohua Ban ${ }^{2 \#}$, Jianxun $\mathrm{Lv}^{1}$, Lin Cheng ${ }^{1}$, Jianmin $\mathrm{Xu}^{3}$, Xinping Shen ${ }^{1}$ \\ ${ }^{1}$ Department of Radiology, The University of Hong Kong-Shenzhen Hospital, Shenzhen, China; ${ }^{2}$ Department of Medical Imaging Center, State Key \\ Laboratory of Oncology in South China, Collaborative Innovation Center for Cancer Medicine, Sun Yat-sen University Cancer Center, Guangzhou, \\ China; ${ }^{3}$ Department of Radiology, Second Clinical Medical College of Jinan University, Shenzhen People's Hospital, Shenzhen, China
}

Contributions: (I) Conception and design: C Zhou, X Ban, X Shen; (II) Administrative support: X Shen; (III) Provision of study materials or patients: All authors; (IV) Collection and assembly of data: C Zhou, X Ban, X Shen; (V) Data analysis and interpretation: C Zhou; (VI) Manuscript writing: All authors; (VII) Final approval of manuscript: All authors.

"These authors contributed equally to this work.

Correspondence to: Xinping Shen. Department of Radiology, The University of Hong Kong-Shenzhen Hospital, 1 Haiyuan Road Futian District, Shenzhen 518000, Guangdong, China. Email: szshenxinping@163.com.

Background: Chromophobe renal cell carcinoma (chRCC) is often confused with oncocytoma and angiomyolipoma without visible fat (AML.wovf). The aim of this study was to determine computed tomography (CT) features predictive of chRCC to distinguish it from oncocytoma and AML.wovf.

Methods: This multicenter study enrolled 38 patients with chRCC, 32 with oncocytoma, and 43 with AML.wovf of the kidney. The clinical and imaging features of all cases were reviewed retrospectively, and associations between the features and histopathology were analyzed using univariate analysis, followed by multinomial logistic regression analysis. Receiver operating characteristic (ROC) curve analysis was used to evaluate logistic regression models and determine optimal cut-off values for numeric data.

Results: Univariate analysis revealed significant differences between chRCC and oncocytoma in tumor ratios of lesion to renal cortex net enhancement (RLRCNE) on both corticomedullary and nephrographic phase images $(\mathrm{P}<0.001$ for both) and calcification $(\mathrm{P}=0.035)$. On multinomial logistic regression analysis, only corticomedullary RLRCNE remained an independent predictor for the differential diagnosis of chRCC from oncocytoma $(\mathrm{P}<0.001)$, with an optimal cut-off value of 0.53. Comparing chRCC and AML.wovf, univariate analysis revealed significant differences in age $(\mathrm{P}=0.003)$, segmental enhancement inversion (SEI) $(\mathrm{P}=0.006)$, corticomedullary RLRCNE $(\mathrm{P}<0.001)$, unenhanced ratio of lesion to renal cortex attenuation (RLRCA; $\mathrm{P}<0.001)$, size $(\mathrm{P}<0.001)$, enhancement pattern over time $(\mathrm{P}=0.017)$, angle $(\mathrm{P}=0.014)$, and central scar $(\mathrm{P}<0.001)$. Only unenhanced RLRCA $(\mathrm{P}<0.001)$, size $(\mathrm{P}=0.003)$, and enhancement pattern over time $(\mathrm{P}=0.002)$ remained as independent predictors on multinomial logistic regression analysis, with optimal cutoff values of 1.13 and $30.9 \mathrm{~mm}$ for RLRCA and size, respectively. On ROC curve analysis of the logistic regression models, the areas under curve (AUC) were 0.888 and 0.963 for chRCC versus oncocytoma and AML.wovf, respectively.

Conclusions: Corticomedullary RLRCNE on CT images was an independent predictor for the differential diagnosis of chRCC from oncocytoma. Unenhanced RLRCA, size, and enhancement pattern over time on CT had predictive value for discriminating chRCC from AML.wovf.

Keywords: Chromophobe renal cell carcinoma (chRCC); predictive features; computed tomography (CT); diagnosis; oncocytoma; angiomyolipoma without visible fat (AML.wovf) 
Submitted Jul 17, 2021. Accepted for publication Dec 06, 2021; Published online: 10 Jan 2022.

doi: 10.21037/qims-21-734

View this article at: https://dx.doi.org/10.21037/qims-21-734

\section{Introduction}

Chromophobe renal cell carcinoma (chRCC) is one of the major malignant kidney tumor types, accounting for 3-5\% of all renal cell carcinoma (RCC) (1). It is thought that chRCC arises from the intercalated cells of the collecting system (2). The CT features of chRCC vary widely, and the CT enhancement characteristics fall between clear cell RCC (ccRCC) and papillary RCC (pRCC) (3). On radiographic images, chRCC can frequently be confused with oncocytoma, and they have a similar pathology (4). Moreover, the enhancement characteristics of angiomyolipoma without visible fat (AML.wovf) on multiphase multidetector CT also fall between those of ccRCC and pRCC (5). Both AML.wovf and non-clear cell RCC (chRCC and pRCC) appear as homogeneous and hyperattenuated masses on unenhanced $\mathrm{CT}$, and undergo both homogeneous and persistent enhancement (6), so it is vital to differentiate between them (7). To date, many studies have investigated the imaging features of chRCC, oncocytoma, and AML.wovf to distinguish chRCCs from oncocytomas (3,7-12). However, some studies have shown inconsistent results $(3,7,8)$ and none of them have compared confounding lesions, such as AML.wovf, with chRCC. In addition, most previous studies have reported absolute attenuation of the same phase, which is variable and associated with the scanner, scanning technique, personal factors, and doses of contrast media used. To reduce these variations, the relative attenuation ratio [i.e., the ratio of lesion to renal cortex attenuation (RLRCA) on unenhanced CT images] and relative net enhancement [i.e., the ratio of lesion to renal cortex net enhancement (RLRCNE) on enhanced phase images] should be used.

Furthermore, renal oncocytoma and AML.wovf are benign, whereas chRCC is malignant. With the aim of early curative surgery for a presumed RCC, some oncocytomas and AML.wovf are being surgically resected without a confirmed diagnosis. To avoid unnecessary surgery in the future, improved medical imaging strategies are needed to preoperatively identify chRCC from benign tumors. In this study, we retrospectively reviewed the clinical and CT features of patients with histologically proven chRCC, oncocytoma, and AML.wovf to identify features that could be used to distinguish chRCC from oncocytoma and AML. wovf. We present the following article in accordance with the STAndards for Reporting of Diagnostic accuracy Studies (STARD) reporting checklist (available at https://qims. amegroups.com/article/view/10.21037/qims-21-734/rc).

\section{Methods}

\section{Patients}

This study was conducted in accordance with the Declaration of Helsinki (as revised in 2013). The study was approved by the institutional review boards of the 2 participating centers and, because of the retrospective nature of the study, the need for informed consent was waived.

All histologically proven consecutive cases of chRCCs, oncocytomas, and AML.wovf at the 2 centers between June 2013 and March 2020 were reviewed. Patients were included if they had a pathologically confirmed diagnosis of chRCC, oncocytoma, or AML.wovf of the kidney and had undergone preoperative multiphase multidetector CT examination of the kidney. Patients were excluded if preoperative multiphase multidetector CT examination of the kidney had not been performed and/or the pathological diagnosis was not clear (e.g., lack of distinction between oncocytoma and chRCC).

\section{CT examinations}

All patients underwent unenhanced and contrast-enhanced CT examinations within the 4 weeks prior to surgery. The CT imaging was performed in 49 patients using a 64-slice spiral CT (Toshiba Aquilion 64; Toshiba Medical Systems, Otawara, Japan) and in the remaining 64 patients using a 128-slice spiral CT (Somatom Definition AS; Siemens Medical Systems, Erlangen, Germany). The scan parameters included a tube voltage of $120 \mathrm{kV}$, variable tube current, field of view of $200-240 \mathrm{~mm}$, pitch of 1.2 , and a matrix of $512 \times 512$. Sagittal, coronal, and axial multiplanar reconstruction images (1-5 mm thick) were obtained with soft tissue kernels. The renal protocol included acquisition of unenhanced images through the kidneys, followed by corticomedullary (30-40 s) and nephrographic (90-120 s) phase images after intravenous administration of iopamiro 
(370 mg I/100 mL; Shanghai Bracco Sine Pharmaceutical Corporation; Shanghai, China) at a dose of $1.0 \mathrm{~mL} / \mathrm{kg}$ body weight and a flow rate of $3 \mathrm{~mL} / \mathrm{s}$.

\section{Imaging analysis}

All images were independently reviewed by 2 experienced radiologists (ZC and SX, with 14 and 33 years experience in diagnostic imaging, respectively) who were blinded to the pathologic information. In the case of discrepancies, final conclusions were reached by consensus. Quantitative data were measured by 2 observers (ZC and SX), mean values were used, and consistency between the 2 observers was calculated. The following radiologic factors were determined: primary tumor size, RLRCA on unenhanced CT images, RLRCNE on images from 2 enhanced phases, tumor shape, location of the tumor center, calcification, segmental enhancement inversion (SEI), enhancement pattern over time, enlarged vessel in the tumor (EVT), central scar, the angle of the parenchymal portion of a mass, and "overflowing beer sign". Tumor size was measured in the maximum dimension in the sagittal, coronal, or axial plane. Attenuation of the tumor and normal renal cortex was measured on unenhanced and all enhanced phase images. The region of interest (ROI; $0.2-1 \mathrm{~cm}^{2}$ ) was placed in the most avidly enhancing parts of enhanced lesions in the corticomedullary phase, with intratumoral calcification and vessels excluded from the ROI. The ROIs of tumors on unenhanced and all enhanced phase images were consistent. Each ROI was measured 3 times and the mean value was used. Then, RLRCA and RLRCNE were calculated. The RLRCNE was calculated as the net enhancement attenuation of the tumor divided by that of the renal cortex. The shape of the lesion was classified as regular or irregular. Regular tumors were those with a round/ovoid shape, whereas irregular tumors were lobulated. Patterns of enhancement were divided into 3 categories: type 1, early washout pattern [corticomedullary net enhancement (CNE) minus nephrographic net enhancement $(\mathrm{NNE}) \geq 10 \mathrm{Hu}$; type 2, prolonged enhancement (CNE minus NNE $<10 \mathrm{Hu}$ ); and type 3, gradual enhancement (NNE minus $\mathrm{CNE} \geq 10 \mathrm{Hu}$ ). The angle of the parenchymal portion of a mass was classified as $\leq 90^{\circ}$ or $>90^{\circ}$. An overflowing beer sign was defined as a contact length between the bulgingout portion of a mass and the adjacent renal capsule of $\geq 3 \mathrm{~mm}$ (13). The SEI was defined as 2 segments within a mass showing different degrees of enhancement on corticomedullary phase imaging, with the relatively highly enhanced segment during the corticomedullary phase becoming less enhanced during the nephrographic phase and the less enhanced segment during the corticomedullary phase becoming highly enhanced during the nephrographic phase, as suggested by Kim et al. (11). An EVT was defined as any vessel in the tumor with a diameter $\geq 2 \mathrm{~mm}$. As per Giambelluca et al. (14), a central scar was defined as a central zone of distinct hypoattenuation during the corticomedullary phase with the bands of hypoattenuation radiating towards the periphery of the lesion, with or without enhancement during the nephrographic phase, and hypo- or iso-attenuation on non-enhanced CT scans.

\section{Statistical analysis}

To determine the diagnostic value of parameters, cases were divided into 3 groups: group 1, with chRCC; group 2, with oncocytoma; and group 3, with AML.wovf. The following clinical and radiologic variables were included in the analysis: gender (male or female), location of the tumor center (extra- or intra-capsular); shape (regular or irregular); calcification (present or absent); SEI (present or absent); enhancement pattern (type 1, 2, or type 3); EVT (present or absent); central scar (present or absent); the angle $\left(\leq 90^{\circ}\right.$ or $>90^{\circ}$ ); and overflowing beer sign (positive or negative). Consistency between observers was assessed by intraclass correlation coefficients.

Univariate analysis was used to compare categorical data using $\chi^{2}$ tests or numeric data using $t$-tests. When the $\mathrm{P}$ value of the numeric data was $<0.05$, cut-off analysis was performed to determine the optimal cut-off value. Then, multiple logistic regression analysis was performed to determine independent predictors. Odds ratios (OR), as estimates of relative risk, with $95 \%$ confidence intervals (CI) were obtained for each risk factor. A 2 -sided $\mathrm{P}<0.05$ was considered statistically significant. Receiver operating characteristic (ROC) curve analysis was used to assess the multinomial logistic regression models obtained.

All analyses were performed using SPSS version 22.0 (IBM Corp., Armonk, NY, USA). The numeric data were show as the mean \pm SD.

\section{Results}

\section{Patient age and gender}

In this study, 38 cases had chRCCs, 32 had oncocytomas, and 43 had AML.wovf. The mean age of the cases at the 
Table 1 Standardized CT imaging data for the different groups according to centers

\begin{tabular}{|c|c|c|c|}
\hline Imaging data & chRCC & Oncocytoma & AML.wovf \\
\hline \multicolumn{4}{|c|}{ Unenhanced RLRCA } \\
\hline Center 1 & $1.16 \pm 0.28$ & $1.11 \pm 0.13$ & $1.34 \pm 0.31$ \\
\hline Center 2 & $1.05 \pm 0.16$ & $1.03 \pm 0.17$ & $1.47 \pm 0.37$ \\
\hline$P$ value & 0.137 & 0.147 & 0.195 \\
\hline Center 1 & $0.49 \pm 0.23$ & $0.76 \pm 0.18$ & $0.57 \pm 0.33$ \\
\hline Center 2 & $0.39 \pm 0.16$ & $0.78 \pm 0.18$ & $0.56 \pm 0.15$ \\
\hline$P$ value & 0.189 & 0.737 & 0.889 \\
\hline \multicolumn{4}{|c|}{ Nephrographic RLRCNE } \\
\hline$P$ value & 0.07 & 0.25 & 0.082 \\
\hline
\end{tabular}

Unless indicated otherwise, data are given as the mean \pm SD. CT, computed tomography; AML.wovf, angiomyolipoma without visible fat; chRCC, chromophobe renal cell carcinoma; RLRCA, ratio of lesion to renal cortex attenuation; RLRCNE, ratio of lesion to renal cortex net enhancement.

time of diagnosis of chRCC, oncocytoma, and AML.wovf was $54.71 \pm 14.01,55.41 \pm 14.59$, and $45.81 \pm 12.50$ years, respectively. Age was found to be associated with a diagnosis of chRCC or AML.wovf $(\mathrm{P}<0.05)$, with an optimal cut-off value of 47 years, but there was no significant difference in age between the chRCC and oncocytoma groups. Moreover, there was no significant difference in the gender distribution among the 3 groups.

\section{CT findings and univariate analysis}

Standardized parameters indicated that there were no significant differences in unenhanced RLRCA or enhanced RLRCNE between the 2 centers (Table 1). The intraclass correlation coefficients for the 2 readers for tumor size, unenhanced RLRCA, corticomedullary RLRCNE, and nephrographic RLRCNE were 0.96, 0.92, 0.95, and 0.93, respectively. The main imaging characteristics of chRCC, oncocytoma, and AML.wovf and the results of univariate analysis are summarized in Table 2. Typical tumors from each of the chRCC, oncocytoma, and AML.wovf groups are shown in Figures 1-3.

The mean lesion size of chRCC, oncocytoma, and AML. wovf was $55.29 \pm 31.22,43.18 \pm 18.83$, and $29.24 \pm 17.42 \mathrm{~mm}$, respectively. Tumor size differed significantly between the chRCC and AML.wovf groups $(\mathrm{P}<0.05)$, with an optimal cut-off value of $30.9 \mathrm{~mm}$; however, there was no significant difference in tumor size between the chRCC and oncocytoma groups. The RLRCNE was significantly lower for chRCCs than oncocytomas on both corticomedullary and nephrographic phase images $(\mathrm{P}<0.001)$, with optimal cut-off values of 0.53 and 0.41 , respectively. The RLRCNE was significantly lower for chRCCs than AML.wovf on corticomedullary phase images $(\mathrm{P}=0.008)$, with an optimal cut-off value of 0.46 , but did not differ significantly between the 2 groups on nephrographic phase images $(\mathrm{P}=0.458)$. The RLRCA on unenhanced CT images did not differ significantly between chRCCs and oncocytoma ( $\mathrm{P}=0.703)$, but was significantly lower for chRCCs than AML.wovf $(\mathrm{P}<0.001)$, with an optimal cut-off value of 1.13 .

Total of 10 (26.3\%) cases with chRCC, 13 (40.5\%) with oncocytoma, and 2 (4.7\%) with AML.wovf showed SEI during the corticomedullary phase and nephrographic phase. Calcifications were present in $13(34.2 \%)$ patients with chRCCs, 4 (12.5\%) patients with oncocytomas, and 7 (16.3\%) cases with AML.wovf. Enhancement pattern type 1 was seen in 21 (55.3\%) cases with chRCC, 24 (75\%) cases with oncocytoma, and $33(76.7 \%)$ cases with AML. wovf. Central scars were present in 11 (28.9\%) cases with chRCC, 14 (43.8\%) cases with oncocytomas, and no cases with AML.wovf. An angle $\leq 90^{\circ}$ was seen in $5(13.2 \%)$ cases with chRCC, 2 (6.3\%) cases with oncocytoma, and 
Table 2 CT and clinical features of patients in the chRCC, oncocytoma, and AML.wovf

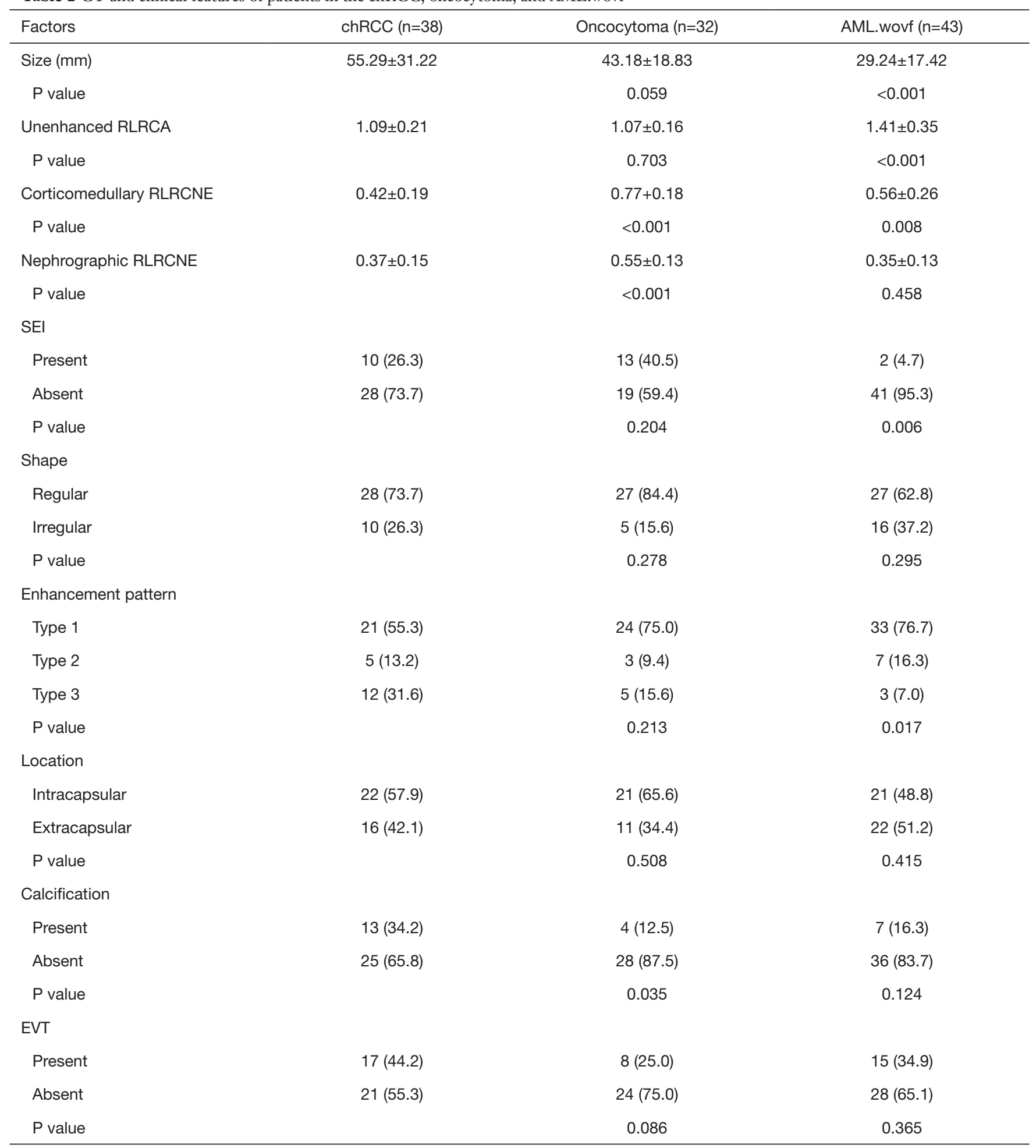

Table 2 (continued) 
Table 2 (continued)

\begin{tabular}{|c|c|c|c|}
\hline Factors & $\operatorname{chRCC}(n=38)$ & Oncocytoma $(n=32)$ & AML.wovf $(n=43)$ \\
\hline$\leq 90^{\circ}$ & $5(13.2)$ & $2(6.3)$ & $16(37.2)$ \\
\hline$>90^{\circ}$ & $33(86.8)$ & $30(93.8)$ & $27(62.8)$ \\
\hline$P$ value & & 0.337 & 0.014 \\
\hline Present & $11(28.9)$ & $14(43.8)$ & $0(0)$ \\
\hline Absent & $27(71.1)$ & $18(56.3)$ & $43(100.0)$ \\
\hline$P$ value & & 0.198 & $<0.001$ \\
\hline \multicolumn{4}{|c|}{ Overflowing beer sign } \\
\hline$P$ value & & 0.104 & 0.160 \\
\hline \multicolumn{4}{|l|}{ Gender } \\
\hline Female & $20(52.6)$ & $24(75.0)$ & $18(41.9)$ \\
\hline Male & $18(47.4)$ & $8(25.0)$ & $25(58.1)$ \\
\hline$P$ value & & 0.054 & 0.332 \\
\hline Age (years) & $54.71 \pm 14.01$ & $55.41 \pm 14.59$ & $45.81 \pm 12.50$ \\
\hline$P$ value & & 0.840 & 0.003 \\
\hline
\end{tabular}

Unless indicated otherwise, data are given as the mean \pm SD or $\mathrm{n}(\%)$. $\mathrm{P}$ values are for comparisons with the chromophobe renal cell carcinoma (chRCC) group. CT, computed tomography; AML.wovf, angiomyolipoma without visible fat; EVT, enlarged vessel in the tumor; RLRCA, ratio of lesion to renal cortex attenuation; RLRCNE, ratio of lesion to renal cortex net enhancement; SEI, segmental enhancement inversion.

16 (37.2\%) cases with AML.wovf. The overflowing beer sign was present in $3(7.9 \%)$ cases with chRCC, none with oncocytoma, and 8 (18.6\%) with AML.wovf.

\section{Predictors for distinguishing chRCC from oncocytoma and AML.wovf}

Univariate analysis revealed that tumor RLRCNE on both corticomedullary and nephrographic phase images and calcification were significantly different between the chRCC and oncocytoma groups $(\mathrm{P}<0.05$; Table 2$)$. In multinomial logistic regression, only RLRCNE on corticomedullary phase images remained an independent predictor for the differential diagnosis of chRCC from oncocytoma (Table 3). Renal tumor with an RLRCNE $<0.53$ on corticomedullary phase images was more likely to be chRCC than oncocytoma (OR $=27.148 ; 95 \%$ CI: 4.908 to $150.155 ; \mathrm{P}<0.001)$, with a sensitivity of $93.8 \%$ and a specificity of $78.9 \%$. Moreover, ROC curve analysis showed that the area under curve (AUC) of the logistic regression model obtained was 0.888 (95\% CI: 0.806 to 0.969), demonstrating that the model is a reasonable predictor for discriminating chRCC from oncocytoma (Figure 4).

Comparing chRCC and AML.wovf groups, univariate analysis revealed significant $(\mathrm{P}<0.05)$ differences in age, SEI, RLRCNE in corticomedullary phase images, RLRCA on unenhanced CT images, size, enhancement pattern, angle, and central scar (Table 2). Multinomial logistic regression analysis showed that only RLRCA on unenhanced CT images, size, and enhancement pattern were independent predictors for discriminating chRCC from AML.wovf (Table 4). Renal tumor with an RLRCA $<1.13$ on unenhanced CT 

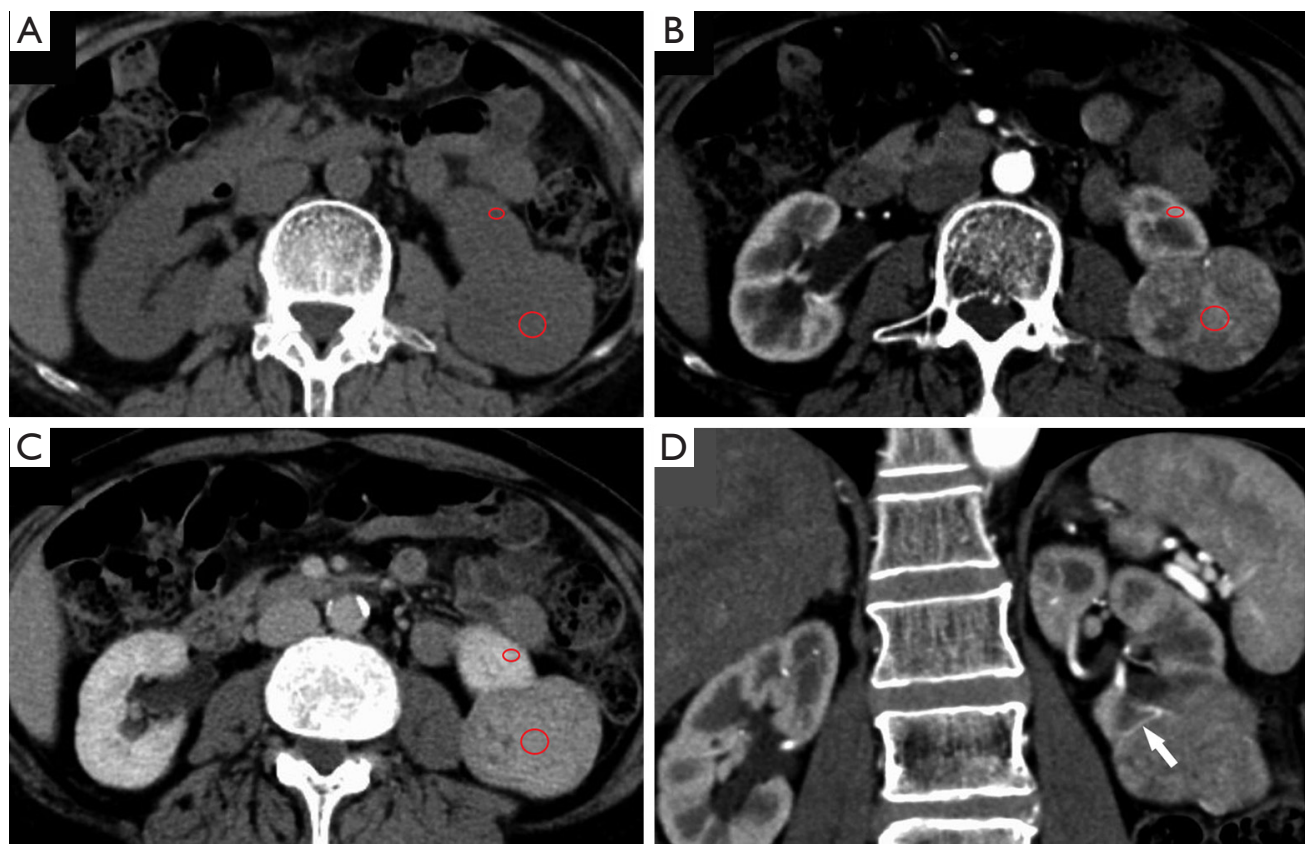

Figure 1 CT images for a 61-year-old woman with irregular chRCC. Representative ROIs (circled) were placed on the tumor and normal renal cortex in each phase. (A) The mean unenhanced attenuation of the lesion and renal cortex was 32 and $30 \mathrm{Hu}$, respectively; the unenhanced RLRCA was 1.07 (32/30). (B) The mean corticomedullary phase attenuation of the lesion and renal cortex was 81 and 148 $\mathrm{Hu}$, respectively. The net enhancement of the lesion and renal cortex during the corticomedullary phase was 49 and $118 \mathrm{Hu}$, respectively; thus, the corticomedullary RLRCNE was 0.42 (49/118). (C) The mean nephrographic phase attenuation of the lesion and renal cortex was 113 and $170 \mathrm{Hu}$, respectively. The net enhancement of the lesion and renal cortex during the nephrographic phase was 81 and $140 \mathrm{Hu}$, respectively; thus, the nephrographic RLRCNE was 0.58 (81/140). (D) Enlarged vessel in the tumor (arrow). CT, computed tomography; ccRCC, chromophobe renal cell carcinoma; RLRCNE, ratio of lesion to renal cortex net enhancement; ROI, region of interest; RLRCA, ratio of lesion to renal cortex attenuation.
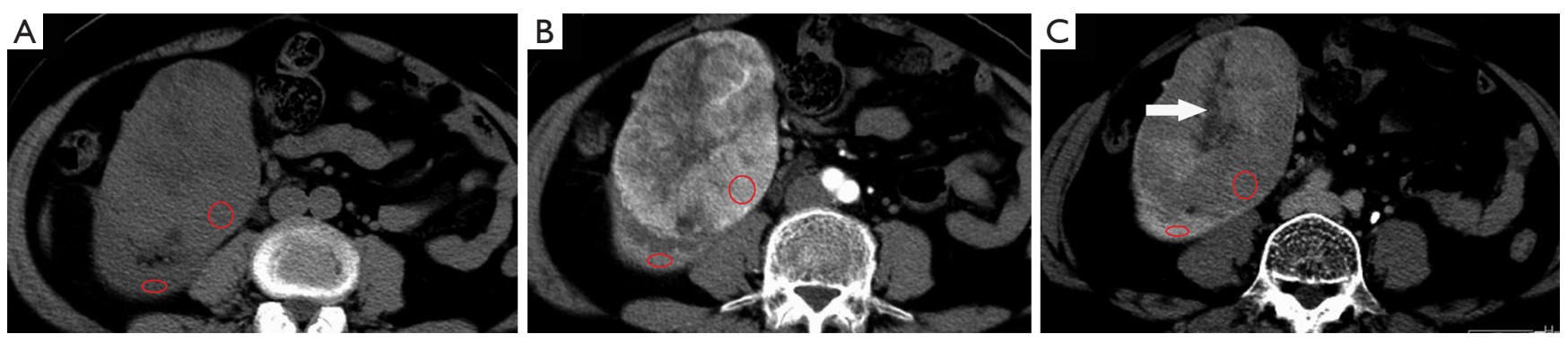

Figure 2 CT images for a 58-year-old woman with irregular oncocytoma. Representative regions of interest (circled) were placed on the tumor and normal renal cortex in each phase. (A) The mean unenhanced attenuation of the lesion and renal cortex was 40 and $30 \mathrm{Hu}$, respectively; the unenhanced RLRCA was 1.33 (40/30). (B) The mean corticomedullary phase attenuation of the lesion and renal cortex was 116 and $129 \mathrm{Hu}$, respectively. The net enhancement of the lesion and renal cortex during the corticomedullary phase was 76 and $99 \mathrm{Hu}$, respectively; thus, the corticomedullary RLRCNE was 0.77 (76/99). (C) The arrow indicates a central scar. The mean nephrographic phase attenuation of the lesion and renal cortex was 103 and $168 \mathrm{Hu}$, respectively. The net enhancement of the lesion and renal cortex during the nephrographic phase was 63 and $138 \mathrm{Hu}$, respectively; thus, the nephrographic RLRCNE was 0.46 (63/138). CT, computed tomography; RLRCNE, ratio of lesion to renal cortex net enhancement; RLRCA, ratio of lesion to renal cortex attenuation. 

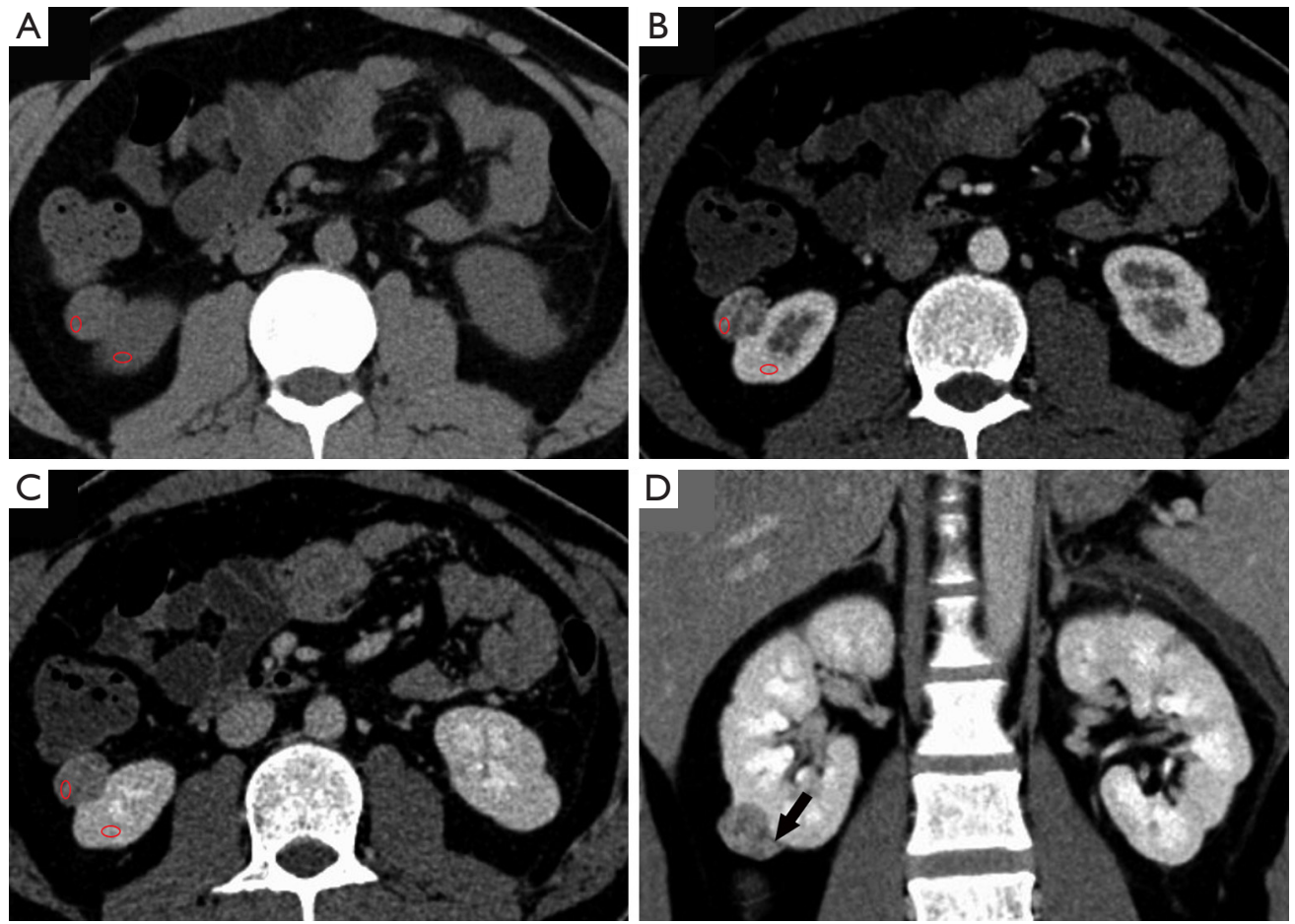

Figure 3 CT images for a 29-year-old man with irregular angiomyolipoma without visible fat. Representative regions of interest (circled) were placed on the tumor and normal renal cortex in each phase. (A) The mean unenhanced attenuation of the lesion and renal cortex was 47 and $35 \mathrm{Hu}$, respectively; the unenhanced RLRCA was 1.34 (47/35). (B) The mean corticomedullary phase attenuation of the lesion and renal cortex was 144 and $220 \mathrm{Hu}$, respectively. (C) The mean nephrographic phase attenuation of the lesion and renal cortex was 99 and 171 $\mathrm{Hu}$, respectively. The net enhancement of lesion and renal cortex on corticomedullary phase were 97 and 185 HU; The corticomedullary RLRCNE (ratios of lesion-to-renal cortex net enhancement) was 0.52 (97/185). The net enhancement of the lesion and renal cortex during the nephrographic phase was 52 and $136 \mathrm{Hu}$, respectively; thus, the nephrographic RLRCNE was 0.38 (52/136). (D) Overflowing beer sign (arrow). CT, computed tomography; RLRCNE, ratio of lesion to renal cortex net enhancement; RLRCA, ratio of lesion to renal cortex attenuation.

Table 3 Multinomial logistic regression analysis of factors distinguishing chromophobe renal cell carcinoma from oncocytoma

\begin{tabular}{lcccc}
\hline Factors & Value & $\beta$ & P value & OR (95\% Cl) \\
\hline Corticomedullary RLRCNE & $<0.53$ & 3.302 & $<0.001$ & $27.148(4.908-150.155)$ \\
Nephrographic RLRCNE & $<0.41$ & 1.127 & 0.146 & $3.087(0.676-14.105)$ \\
Calcification & Present & 0.792 & 0.359 & $2.207(0.406-11.999)$ \\
\hline
\end{tabular}

$\mathrm{Cl}$, confidence interval; OR, odds ratio; RLRCA, ratio of lesion to renal cortex attenuation; RLRCNE, ratio of lesion to renal cortex net enhancement.

images was more likely to be chRCC than AML.wovf (OR $=34.271$; 95\% CI: 4.047-290.211; $\mathrm{P}<0.05$ ), with a sensitivity of $81.4 \%$ and a specificity of $60.5 \%$. Moreover, a renal tumor $\geq 30.9 \mathrm{~mm}$ in size was more likely to be chRCC than AML.wovf (OR =19.021; 95\% CI: 2.692-134.381; $\mathrm{P}<0.05$ ), with a sensitivity of $81.6 \%$ and a specificity of $74.4 \%$.
Furthermore, compared with the type 1 enhancement pattern, a renal tumor with a type 3 enhancement pattern was more likely to be chRCC than AML.wovf $(\mathrm{OR}=18.940$; 95\% CI: $1.587-226.029 ; \mathrm{P}<0.05)$, with a sensitivity of $31.6 \%$ and a specificity of $93.0 \%$. The AUC of the logistic regression model obtained was 0.963 (95\% CI: $0.928-$ 
0.997), indicating that the model was a good predictor for discriminating chRCC from AML.wovf (Figure 4). Combined evaluation of RLRCA $<1.13$ on unenhanced CT images, tumor size $\geq 30.9 \mathrm{~mm}$, and type 3 enhancement pattern had a sensitivity of $97.4 \%$ and a specificity of $81.4 \%$.

\section{Discussion}

For the characterization of renal masses, CT remains the first-line imaging examination. The CT features of chRCCs vary widely, and chRCCs remain challenging to diagnose. In this study, we compared the clinical and CT features

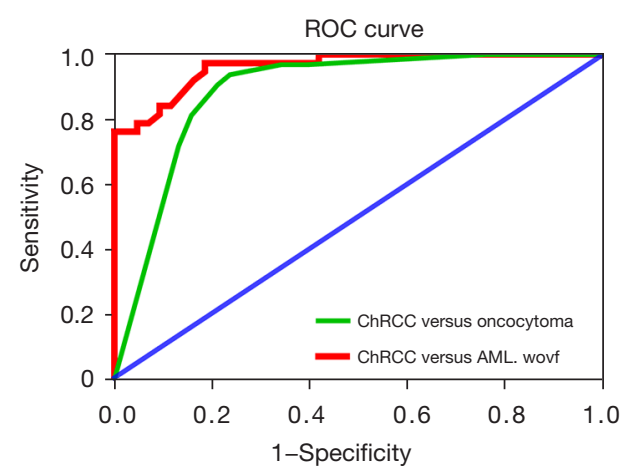

Figure 4 ROC curves of the 2 multinomial logistic regression models. The AUC for the chRCC versus oncocytoma and the chRCC versus AML.wovf logistic regression models were 0.888 and 0.963 , respectively. ROC, receiver operating characteristic; ccRCC, chromophobe renal cell carcinoma; AML.wovf, angiomyolipoma without visible fat; AUC, area under the curve. of 38 chRCCs with those of 32 oncocytomas and 43 AML.wovf to determine predictors that could help in the differential diagnosis of chRCC. Clinically, the mean age of chRCC patients at the time of diagnosis was 54.71 years in the present study, which is consistent with some previous reports $(7,15)$, but a little younger than that in other studies $(3,16)$. In the present study, the mean age of chRCC patients was not significantly different to that of those with oncocytoma, but was significantly greater than that of those with AML.wovf. In addition, $55.8 \%$ of chRCC patients were female in the present study, which is higher than $40 \%$ and $33 \%$ female chRCC patients in previous reports, respectively $(3,7)$, but there was no significant difference in the gender distribution among our 3 groups.

The results of previous studies have been inconsistent. For example, chRCC has been reported to have greater homogeneity and the presence of a central scar and calcification (3). Another study reported that chRCC tended to be more homogeneous than oncocytomas, which can be heterogeneous, but that there was considerable overlap in other features (e.g., scar, SEI) between tumors (16). There has been SEI observed in both renal oncocytoma and chRCC, with no significant difference between the 2 $(9,17)$, and the same has been reported for central scars (17). However, CT imaging features such as calcifications, stellate scar, and SEI are more common in oncocytoma, and they may help differentiate oncocytoma from chRCC (8). The wide spectrum of imaging findings for renal oncocytoma is associated with differences in tumor cellularity (18). In the present study, we not only analyzed a wider range of CT features (tumor size, RLRCA, RLRCNE, shape, location,

Table 4 Multinomial logistic regression analysis of factors distinguishing chromophobe renal cell carcinoma fromangiomylipoma without visible fat

\begin{tabular}{lcccc}
\hline Factors & Value & $\beta$ & P value & OR (95\% Cl) \\
\hline Unenhanced RLRCA & $<1.13$ & 3.534 & 0.001 & $34.271(4.047-290.211)$ \\
Corticomedullary RLRCNE & $>0.46$ & 1.121 & 0.294 & $3.067(0.378-24.884)$ \\
SEI & Absent & -2137 & 0.129 & $0.118(0.007-1.864)$ \\
Size & $\geq 30.9 \mathrm{~mm}$ & 2.946 & 0.003 & $19.021(2.692-134.381)$ \\
Age & $\geq 47$ years & 1.569 & 0.124 & $4.804(0.649-35.542)$ \\
Enhancement pattern & Type 3 & 2.941 & 0.020 & $18.940(1.587-226.029)$ \\
& Type 2 & -1.262 & 0.400 & $0.283(0.015-5.330)$ \\
Angle & $\leq 90^{\circ}$ & -1.771 & 0.116 & $0.170(0.019-1.544)$ \\
Central scar & Absent & -18.751 & 0.999 & $0.000(0.000-)$ \\
\hline
\end{tabular}

$\mathrm{Cl}$, confidence interval; OR, odds ratio; RLRCA, ratio of lesion to renal cortex attenuation; RLRCNE, ratio of lesion to renal cortex net enhancement. 
growth pattern, calcification, SEI, contrast enhancement pattern, EVT, angle, overflowing beer sign, and central scar), but also used a new parameter (RLRCNE) to standardize the enhancement measurement. Nonetheless, only RLRCNE in the enhanced corticomedullary and nephrographic phase images and calcification were related to chRCC and oncocytoma in univariate analysis. The RLRCNE in the images from the 2 enhanced phases was lower in chRCC than oncocytoma, which is consistent with previous studies which have reported that enhancement was lower in chRCC than oncocytoma during all phases $(7,8,11)$. In the present study, calcifications were more common in chRCC than oncocytoma, which is not consistent with a previous report (8). Furthermore, only corticomedullary RLRCNE remained an independent predictor of the differential diagnosis of chRCC from oncocytoma in multinomial logistic regression. In particular, a renal tumor with corticomedullary RLRCNE $<0.53$ was more likely to be chRCC than oncocytoma (OR $=27.148 ; 95 \%$ CI: 4.908 to $150.155 ; \mathrm{P}<0.05)$. In addition, ROC curve analysis showed that the AUC of the logistic regression model was 0.888 , demonstrating that the model was a reasonable predictor for the discrimination of chRCC from oncocytoma.

To further confirm the diagnosis of chRCC, we compared the clinical and imaging features of chRCC with those of AML.wovf. On CT imaging, AML.wovf can appear as a small renal mass with homogeneous hyperattenuation without calcification (19). Calcification generally does not occur in AML.wovf $(6,19,20)$. The presence of an overflowing beer sign or an angle $\leq 90^{\circ}$ are independent imaging parameters to differentiate AML.wovf from RCC (13). However, in the present study, calcification was present in $8(16.3 \%)$ cases with AML.wovf and the overflowing beer sign was present in $3(7.9 \%)$ cases with chRCC, and there was no significant difference in either parameter between the chRCC and AML.wovf groups. In the present study, age, SEI, corticomedullary RLRCNE, unenhanced RLRCA, size, enhancement pattern, angle, and central scar were shown to differ significantly between the 2 groups in univariate analysis $(\mathrm{P}<0.05)$. The chRCC can be hyperattenuated on unenhanced CT; in the present study, the mean unenhanced RLRCA was 1.09 . To date, no acceptable threshold level of attenuation has been described on unenhanced CT to reliably differentiate AML.wovf from RCC (6). In the present study, the RLRCA on unenhanced CT images was significantly lower for chRCC than AML. wovf, and was an independent predictor for the differential diagnosis of chRCC on multinomial logistic regression analysis $(\mathrm{P}<0.05)$ with an optimal cut-off value of 1.13 . A renal tumor with an RLRCA $<1.13$ on unenhanced CT images was more likely to be chRCC than AML.wovf (OR $=34.271 ; 95 \%$ CI: 4.047-290.211; $\mathrm{P}<0.05)$. In addition, most chRCCs are large at the time of diagnosis (21). In the present study, the size of chRCCs was larger than that of AML.wovf, and size was a predictor for distinguishing chRCC from AML.wovf, with an optimal cut-off value of $30.9 \mathrm{~mm}$. Interestingly, renal tumors $\geq 30.9 \mathrm{~mm}$ are more likely to be chRCC than AML.wovf $(\mathrm{OR}=19.021$; 95\% CI: 2.692-134.381; $\mathrm{P}<0.05)$. Moreover, compared with the type 1 enhancement pattern, renal tumors with a type 3 enhancement pattern are more likely to be chRCC than AML.wovf (OR =18.940; 95\% CI: 1.587-226.029; $\mathrm{P}<0.05)$. The AUC of the logistic regression model was 0.963 , indicating that the model was a good predictor for discriminating chRCC from AML.wovf.

The present study has several limitations. First, because of the retrospective nature of this study, the tumors were not scanned using the same scanner with the same CT parameters. Second, because multinomial logistic regression analysis requires a large number of patients, the CT data used in this study came from 2 centers. Nevertheless, the slice thickness of CT images for all cases in this series was 1-2 $\mathrm{mm}$, which may be acceptable for morphologic imaging analysis. Moreover, we used RLRCNE and RLRCA instead of absolute attenuation to standardize these parameters, with no significant differences between the 2 centers. Third, the 3 most common subtypes of RCC are chRCC, ccRCC, and pRCC, but we did not include ccRCC and pRCC in the present study. However, according to previous studies $(3,5,22)$, the enhancement characteristics of chRCCs, oncocytomas, and AML.wovf arebeing less than those of ccRCC and more than those of pRCC. Among the 3 RCC subtypes (i.e., ccRCC, chRCC, and pRCC), chRCC is the most difficult to differentiate from oncocytomas and AMLs. As such, we analyzed the features of the 3 kinds of tumors to find predictive features to distinguish chRCC from oncocytoma and AML.wovf. The differential diagnosis of ccRCC and pRCC from the 2 common benign tumors requires further investigation. In addition, quantitative spectral CT with a stretched-exponential non-linear regression analysis model may enhance the ability to differentially diagnose renal tumors (23). Further prospective studies using quantitative spectral CT may provide additional information regarding differential diagnosis. 
In conclusion, $\mathrm{CT}$ features had predictive value for discriminating chRCC from oncocytoma and AML.wovf. Renal tumors with corticomedullary RLRCNE $<0.53$ were more likely to be chRCC than oncocytoma. Renal tumors with RLRCA $<1.13$ on unenhanced CT images, and/or size $\geq 30.9 \mathrm{~mm}$, and/or a type 3 enhancement pattern were more likely to be chRCC than AML.wovf.

\section{Acknowledgments}

Funding: None.

\section{Footnote}

Reporting Checklist: The authors have completed the STARD reporting Checklist. Available at https://qims.amegroups. com/article/view/10.21037/qims-21-734/rc

Conflicts of Interest: All authors have completed the ICMJE uniform disclosure form (available at https://qims. amegroups.com/article/view/10.21037/qims-21-734/coif). The authors have no conflicts of interest to declare.

Ethical Statement: The authors are accountable for all aspects of the work in ensuring that questions related to the accuracy or integrity of any part of the work are appropriately investigated and resolved. The study was conducted in accordance with the Declaration of Helsinki (as revised in 2013). This retrospective study was approved by the Ethics Committee of the 2 centers, and the need for informed consent was waived.

Open Access Statement: This is an Open Access article distributed in accordance with the Creative Commons Attribution-NonCommercial-NoDerivs 4.0 International License (CC BY-NC-ND 4.0), which permits the noncommercial replication and distribution of the article with the strict proviso that no changes or edits are made and the original work is properly cited (including links to both the formal publication through the relevant DOI and the license). See: https://creativecommons.org/licenses/by-nc-nd/4.0/.

\section{References}

1. Warren AY, Harrison D. WHO/ISUP classification, grading and pathological staging of renal cell carcinoma: standards and controversies. World J Urol 2018;36:1913-26.

2. Störkel S, Steart PV, Drenckhahn D, Thoenes W.
The human chromophobe cell renal carcinoma: its probable relation to intercalated cells of the collecting duct. Virchows Arch B Cell Pathol Incl Mol Pathol 1989;56:237-45.

3. Raman SP, Johnson PT, Allaf ME, Netto G, Fishman EK. Chromophobe renal cell carcinoma: multiphase MDCT enhancement patterns and morphologic features. AJR Am J Roentgenol 2013;201:1268-76.

4. Cornelis F, Tricaud E, Lasserre AS, Petitpierre F, Bernhard JC, Le Bras Y, Yacoub M, Bouzgarrou M, Ravaud A, Grenier N. Routinely performed multiparametric magnetic resonance imaging helps to differentiate common subtypes of renal tumours. Eur Radiol 2014;24:1068-80.

5. Kim SH, Kim CS, Kim MJ, Cho JY, Cho SH. Differentiation of Clear Cell Renal Cell Carcinoma From Other Subtypes and Fat-Poor Angiomyolipoma by Use of Quantitative Enhancement Measurement During ThreePhase MDCT. AJR Am J Roentgenol 2016;206:W21-8.

6. Park BK. Renal Angiomyolipoma Based on New Classification: How to Differentiate It From Renal Cell Carcinoma. AJR Am J Roentgenol 2019;212:582-8.

7. Akın IB, Altay C, Güler E, Çamlıdağ İ, Harman M, Danacı M, Tuna B, Yörükoğlu K, Seçil M. Discrimination of oncocytoma and chromophobe renal cell carcinoma using MRI. Diagn Interv Radiol 2019;25:5-13.

8. Wu J, Zhu Q, Zhu W, Chen W, Wang S. Comparative study of CT appearances in renal oncocytoma and chromophobe renal cell carcinoma. Acta Radiol 2016;57:500-6.

9. Potretzke AM, Potretzke TA, Bauman TM, Knight BA, Park AM, Mobley JM, Figenshau RS, Siegel CL. Computed Tomography and Magnetic Resonance Findings of Fat-Poor Angiomyolipomas. J Endourol 2017;31:119-28.

10. Schieda N, McInnes MD, Cao L. Diagnostic accuracy of segmental enhancement inversion for diagnosis of renal oncocytoma at biphasic contrast enhanced CT: systematic review. Eur Radiol 2014;24:1421-9.

11. Kim JI, Cho JY, Moon KC, Lee HJ, Kim SH. Segmental enhancement inversion at biphasic multidetector CT: characteristic finding of small renal oncocytoma. Radiology 2009;252:441-8.

12. Woo S, Cho JY, Kim SH, Kim SY. Comparison of segmental enhancement inversion on biphasic MDCT between small renal oncocytomas and chromophobe renal cell carcinomas. AJR Am J Roentgenol 2013;201:598-604.

13. Kim YH, Han K, Oh YT, Jung DC, Cho NH, Park SY. Morphologic analysis with computed tomography may 
help differentiate fat-poor angiomyolipoma from renal cell carcinoma: a retrospective study with 602 patients. Abdom Radiol (NY) 2018;43:647-54.

14. Giambelluca D, Pellegrino S, Midiri M, Salvaggio G. The "central stellate scar" sign in renal oncocytoma. Abdom Radiol (NY) 2019;44:1942-3.

15. Vera-Badillo FE, Conde E, Duran I. Chromophobe renal cell carcinoma: a review of an uncommon entity. Int J Urol 2012;19:894-900.

16. Schieda N, Lim RS, McInnes MDF, Thomassin I, Renard-Penna R, Tavolaro S, Cornelis FH. Characterization of small $(<4 \mathrm{~cm})$ solid renal masses by computed tomography and magnetic resonance imaging: Current evidence and further development. Diagn Interv Imaging 2018;99:443-55.

17. Rosenkrantz AB, Hindman N, Fitzgerald EF, Niver BE, Melamed J, Babb JS. MRI features of renal oncocytoma and chromophobe renal cell carcinoma. AJR Am J Roentgenol 2010;195:W421-7.

18. Ishigami K, Jones AR, Dahmoush L, Leite LV, Pakalniskis MG, Barloon TJ. Imaging spectrum of renal oncocytomas: a pictorial review with pathologic correlation. Insights Imaging 2015;6:53-64.

Cite this article as: Zhou C, Ban X, Lv J, Cheng L, Xu J, Shen X. Role of computed tomography features in the differential diagnosis of chromophobe renal cell carcinoma from oncocytoma and angiomyolipoma without visible fat. Quant Imaging Med Surg 2022;12(4):2332-2343. doi: 10.21037/ qims-21-734
19. Lim RS, Flood TA, McInnes MDF, Lavallee LT, Schieda $\mathrm{N}$. Renal angiomyolipoma without visible fat: Can we make the diagnosis using CT and MRI? Eur Radiol 2018;28:542-53.

20. Schieda N, Kielar AZ, Al Dandan O, McInnes MD, Flood TA. Ten uncommon and unusual variants of renal angiomyolipoma (AML): radiologic-pathologic correlation. Clin Radiol 2015;70:206-20.

21. Shuch B, Amin A, Armstrong AJ, Eble JN, Ficarra V, Lopez-Beltran A, Martignoni G, Rini BI, Kutikov A. Understanding pathologic variants of renal cell carcinoma: distilling therapeutic opportunities from biologic complexity. Eur Urol 2015;67:85-97.

22. Young JR, Margolis D, Sauk S, Pantuck AJ, Sayre J, Raman SS. Clear cell renal cell carcinoma: discrimination from other renal cell carcinoma subtypes and oncocytoma at multiphasic multidetector CT. Radiology 2013;267:444-53.

23. Yang X, Sun W, Huang D, Li H, Zhao Y, Li P, Liu Y. Quantitative spectral CT evaluation of kidney tumors with the stretched-exponential nonlinear regression analysis model. Quant Imaging Med Surg 2021;11:676-84. 